\title{
TOPICAL USE OF TERRAMYCIN OINTMENT IN TRACHOMA*
}

BY

\section{R. NACCACHE}

Beirut, Lebanon

Methods.-Throughout this investigation we used ophthalmic tubes each containing $\frac{1}{8} \mathrm{Oz}$. of a 0.50 per cent. terramycin ointment. $\dagger$ The treatment was followed for 3 months in each case.

Material.-The patients were unselected; all the cases of trachoma who attended the Lebanese Red Cross Eye Clinic of Moussaitbeh were included, provided:

(a) they did not suffer from any other ophthalmic or debilitating disease;

(b) they attended the clinic regularly;

(c) they had not had other treatment for trachoma for at least 6 months;

(d) they used the ointment given to them regularly, twice a day.

Classification.-The patients were classified into four groups, according to MacCallan (1936). Only cases of Trachoma I, II, and III were treated. We did not follow MacCallan's sub-divisions of Trachoma I and II because:

(i) We found that cases of Trachoma I of the general lymphocytic type were difficult to diagnose clinically in the absence of laboratory facilities. Thus only the follicular type of Trachoma I, which is much more frequently met with in Lebanon, was considered.

(ii) We found that the sub-division of Trachoma II into (a) the follicular type, and (b) the papillary hypertrophic type was too theoretical. Most often our patients exhibit a mixture of these changes with predominance of one or the other. We tried to record the type of trachoma according to the follicles or papillary hypertrophy present.

(iii) Pannus was recorded as follows:

$(+)$-up to $\frac{1}{4}$ of the cornea,

$(++)$ - more than $\frac{1}{4}$ of the cornea.

$(+++)$-pannus reaching pupil (patient's head illuminated by examination lamp).

\section{Results}

36 patients were treated without side-effects, or toxic or allergic reactions.

Trachoma I.-Six cases were treated, one male and five females, all under the age of 8. A clinical cure without scars was obtained in all of them.

Trachoma II.-Twenty-two cases were treated, fourteen males and eight females. This series comprised eleven cases of the follicular type including six cases of early Trachoma II, and eleven cases of mixed follicular and papillary hypertrophic type.

(a) A clinical cure was obtained in seven of the eleven follicular cases. Pannus was greatly reduced but did not disappear.

(b) A cure was obtained only in two of the eleven cases of mixed type with predominance of papillary hypertrophy (Figs $1 a$ and $b$ and $2 a$ and $b$ opposite). The rest showed great improvement. Scar formation in this group was more apparent. 


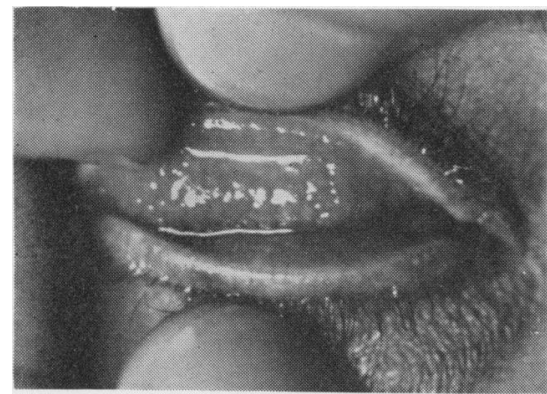

FIG. 1(a).-Case 35. Trachoma II with marked papillary hypertrophy. Right eye.

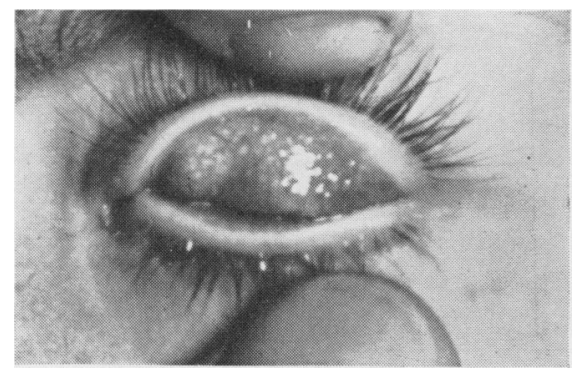

FIG. 2(a).-Case 34. Trachoma II with numerous well defined follicles. Right eye.

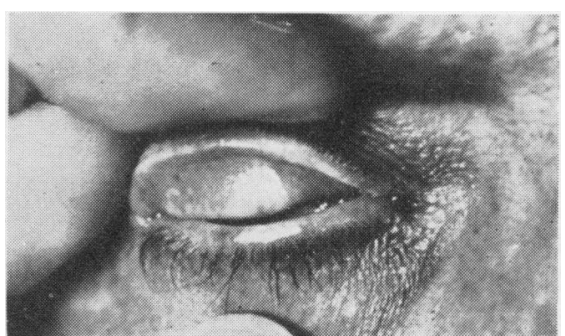

FIG. 1(b).-Same eye after 5 weeks terramycin therapy, showing flattening of papillary hypertrophy and scarring at upper edge of tarsus.

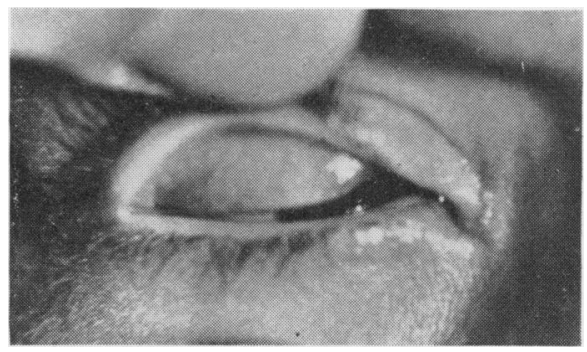

FIG. 2(b).-Dame eye after 5 weeks terramycin therapy, showing decrease in number of follicles and fine criss-cross scars especially at temporal angle. The remaining follicles have lost their sharp edges and are flatter.

Trachoma III.-Eight cases were treated, all females. They showed some improvement, mainly subjective, but none was cured.

\section{Comments}

This investigation led to the following conclusions regarding terramycin:

(1) It is an effective drug against the secondary infection which plays such an important role in the evolution of the disease in the Middle East. This action is shown by the rapid subjective and objective improvement during the first week of therapy, especially in Trachoma II of the papillary type.

(2) It is a potent anti-trachomatous agent. This is shown by the disappearance of the follicles in cases of Trachoma I and early Trachoma II where there was a minimum of secondary infection.

The effect of terramycin was found to vary according to several factors:

(a) Duration of Disease.-The improvement was greater in recent cases.

(b) Grade of Trachoma.-Trachoma I and early Trachoma II were more sensitive, Trachoma III was more resistant.

(c) Type of Trachoma.-The response of the papillary hypertrophy was better at the beginning of the treatment, but from the 4 th to 6 th week a stationary condition 
was reached, whereas the follicles which responded slowly at first continued to decrease.

(d) Degree of Involvement of Tarsus.-As one might expect, cases with an infected, thick, and distorted tarsus responded poorly to terramycin.

(e) Age of Patient.-Younger patients responded better than older ones, other things being equal. Up to 20 years of age the response was generally good.

Our results, though not so brilliant as those of Mitsui and others (1951) seem to be better than those obtained by Siniscal (1952). We believe that the classification used by Mitsui (1952) explains why this author obtained clinical cures in such a short period as 2 or 3 weeks. Cases which this author describes as acute trachoma belong by MacCallan's classification to the general lymphocytic type of Trachoma I. These cases represent the earliest clinical stage of trachomatous infection and are rarely met with in Middle East eye clinics. Patients in Lebanon report to clinics only when lacrimation, photophobia, sensation of foreign body, or discharge become troublesome. All these symptoms are largely due to secondary infection and indicate a certain duration of evolution in the trachoma.

We agree with Siniscal (1952) when he states that trachoma and its response to therapy vary to a great extent according to the country, climate, race, and habits of patients. This might be the reason why terramycin acted only on secondary infection in his patients.

In our cases, the best results were obtained in cases of Trachoma I which were found during a routine examination of symptom-free and apparently healthy children, in whom secondary infection could be practically eliminated.

\section{Conclusion}

Terramycin is a very valuable drug for the treatment of trachoma. It has the advantage over sulfonamides in quicker action, and the absense of toxicity permits longer treatment, especially in cases of children, pregnant women, and debilitated patients.

Mechanical, caustic, or surgical treatments may still be indicated in stubborn cases or in those with deep involvement of the tarsus, but only after an extensive trial of terramycin.

\section{Summary}

Thirty-six cases of trachoma were treated during a period of 3 months by local application of a 0.50 per cent. terramycin ointment. Six cases of Trachoma I were cured; seven cases of early Trachoma II were cured; fifteen cases of Trachoma-II were greatly improved; eight cases of Trachoma III were moderately improved.

Terramycin was found to be a potent drug against secondary infection and trachomatous lesions.

\section{REFERENCES}

MacCallan, A. F. (1936). “ Trachoma ". Butterworth, London.

Mitsu, Y. (1952). J. méd. liban., 5, 203. TANAKA, C., TOYA, H., IWASHIGE, Y., and Yamashita, K. (1951). Arch. Ophthal.,
Chicago, 46, 235.

Siniscal, A. A. (1952). Amer. J. Ophthal., 35, 671-683. 